\title{
Liquid Pre-Freezing Percolation Transition to Equilibrium Crystal-in-Liquid Mesophase
}

\author{
Leslie V. Woodcock \\ Department of Physics, University of Algarve, Faro, Portugal \\ Correspondence to: Leslie V. Woodcock, vwoodcock@ualg.pt \\ Keywords: Liquid State, Percolation, Phase Transition, Pre-Freezing Mesophase \\ Received: May 25, 2018 Accepted: June 23, 2018 Published: July 23, 2018 \\ Copyright $\odot 2018$ by authors and Scientific Research Publishing Inc. \\ This work is licensed under the Creative Commons Attribution International License (CC BY 4.0). \\ http://creativecommons.org/licenses/by/4.0/

\section{(c) (1) Open Access}

\section{ABSTRACT}

Pre-freezing anomalies are explained by a percolation transition that delineates the existence of a pure equilibrium liquid state above the temperature of $1^{\text {st }}$-order freezing to the stable crystal phase. The precursor to percolation transitions are hetero-phase fluctuations that give rise to molecular clusters of an otherwise unstable state in the stable host phase. In-keeping with the Ostwald's step rule, clusters of a crystalline state, closest in stability to the liquid, are the predominant structures in pre-freezing hetero-phase fluctuations. Evidence from changes in properties that depend upon density and energy fluctuations suggests embryonic nano-crystallites diverge in size and space at a percolation threshold, whence a colloidal-like equilibrium is stabilized by negative surface tension. Below this transition temperature, both crystal and liquid states percolate the phase volume in an equilibrium state of dispersed coexistence. We obtain a preliminary estimate of the prefreezing percolation line for water determined from higher-order discontinuities in Gibbs energy that derivatives the isothermal rigidity $\left[(\mathrm{dp} / \mathrm{d} \rho)_{\mathrm{T}}\right]$ and isochoric heat capacity $[(\mathrm{dU} / \mathrm{dT}) \mathrm{v}]$ respectively. The percolation temperature varies only slightly with pressure from $51.5^{\circ} \mathrm{C}$ at 0.1 $\mathrm{MPa}$ to around $60^{\circ} \mathrm{C}$ at $100 \mathrm{MPa}$. We conjecture that the predominant dispersed crystal structure is a tetrahedral ice, which is the closest of the higher-density ices (II to XV) to liquid water in configurational energy. Inspection of thermodynamic and transport properties of liquid argon also indicate the existence of a similar prefreezing percolation transition at ambient pressures $(0.1 \mathrm{MPa})$ around $90 \mathrm{~K}, \sim 6 \%$ above the triple point $(84 \mathrm{~K})$. These findings account for many anomalous properties of equilibrium and supercooled liquids generally, and also explain Kauzmann's "paradox" at a "glass" transition.

\section{INTRODUCTION}

In thermodynamic equilibrium between two coexisting phases, the surface tension is positive and the 
first-order phase transition is characterized by phase separation to minimize the excess free energy of the interface. Change of mole fraction, in accord with the lever rule, is characterized with a latent heat, change in molar enthalpy at constant temperature and a latent work, change in molar volume at constant pressure. If the surface tension, and hence also interfacial excess Gibbs energy, were to be negative, first-order 2-phase coexistence would not be possible as the two states would spontaneously disperse to a colloidal state. This is precisely what has recently been shown to happen, however, above the critical temperature between the limits of existence of gas and liquid states which are delineated by percolation transition loci [1-3].

Pre-transition changes in thermodynamic properties are well documented, and generally explained by an effect known as "heterophase fluctuations", with a long history of experimental investigation and literature discussion [4]. The effect is manifested by a mathematical bifurcation of $2^{\text {nd }}$ order thermodynamic properties, i.e. $2^{\text {nd }}$ derivatives of Gibbs energy with respect to temperature or pressure, heat capacity and compressibility respectively, in the vicinity of the transition to the more stable phase. It is based on the idea that the macroscopic transition of a substance from one phase to another begins with an increased presence of small nuclei of the new phase within the host old phase equilibrium state as the transition is approached. These micro-crystallites can develop from spontaneous equilibrium density fluctuations which are manifestations of a generalized statistical equilibrium in which they may be seen as dispersed hetero-phase particles, with the host phase considered as quasi-solvent [5]. This general description of gas-liquid equilibria leads to a quantitative explanation of a constant minimum of the isothermal rigidity, for example, in the delineation of supercritical gaseous and liquid states of existence [1-5].

At a crystal-liquid interface, unlike a vapor-liquid interface, the perpendicular diagonal element of the pressure tensor has a gradient that, besides differing from the parallel pressures, is not spherically symmetric. The surface tension of any crystal-liquid interface $\left(\gamma_{c l}\right)$ depends upon the orientational plane of the crystal. If the Miller indices describing a crystalface in the $\mathrm{x}-\mathrm{y}$ plane, are $i, j, k$, it can generally be defined by an ensemble average that can be integrated across the interfacial region to obtain a surface tension.

$$
\gamma_{c l}(i, j, k)=\int<p_{z z}-1 / 2\left(p_{x x}+p_{y y}\right)>d z
$$

Surface tensions, or equivalently, excess surface free energy per unit area, may be positive, or negative for a crystal face when $p_{z z}(z)<1 / 2\left(p_{x x}+p_{y y}\right)$, on average. For all interfaces between two thermodynamically coexisting states of one component in a 2-phase region of $1^{\text {st }}$-order phase coexistence, $\gamma_{c l}$ must be positive. The total Gibbs energy of the whole system is a minimum when the surface area is the minimized. When crystal and liquid phases coexist in a $1^{\text {st }}$-order phase transition, there can be no, face, i.e. no i, $j, k$ set, for which the surface tension is negative, otherwise, the crystal phase would be unstable with respect to a spontaneous dispersion to a colloidal-like state. This is not necessarily the case with alternative crystal structures, however.

In the simple classical nucleation theory, Gibbs energy, and hence also the thermodynamic stability, of a small crystalline cluster of atoms or molecules, are simply expressed in terms of bulk thermodynamic quantities, notably, the chemical potentials $(\mu)$ of the respective phases and the excess interfacial Gibbs energy per unit area $\left(\Delta G^{e}\right)$ when T and $p$ are the fixed state variables. Then the relative Gibbs energy of a crystalline cluster of $n$ atoms is

$$
\Delta G^{e}(n)=\gamma_{c l} A(n)-n\left(\mu_{l}-\mu_{c}\right)
$$

where the area $A(n)=\xi \rho_{c}^{-2 / 3} n^{2 / 3}: \xi$ is constant depending upon shape of crystallite. At supercooled temperatures $T<T_{f}, \mu_{c}<\mu_{l}$, hence the surface tension between phases in a first-order phase transition must be positive. Therefore $\Delta G^{e}(n)$ passes through a positive maximum, known as the "free energy barrier to nucleation", before decreasing to become negative, thereby stabilizing the embryonic crystalline state approaching $\mu_{c}(T)$ for large $n$.

For an equilibrium one-component liquid at temperatures above $T_{f}$ however, there can be no hetero-phase fluctuations leading to nano-crystallites of the most stable phase with a first-order transition, because they would be unstable when $\gamma_{c l}$ is positive. According to Ostwalds step rule [6], the prominent fluctuations will lead to crystallites of the least stable crystal phases but perhaps closer entropy to the liq- 
uid, thereby minimizing overall entropy production in any real crystallization process [7]. It is well known that for many crystal surfaces in contact with a fluid, the surface tensions must be negative. For example, catalysis of many gas phase reactions on crystal surfaces could not happen unless the surface tension is negative. We can expect the crystal structures nearest in stability to the liquid to exhibit stable hetero-phase fluctuations. These stable crystalline clusters increase with decreasing temperature, and eventually percolate the phase volume to give a colloidal me so-phase in thermodynamic equilibrium at temperatures above freezing mesophase.

Accordingly, in the simple homogeneous nucleation model of Equation (2), if the liquid is in thermodynamic equilibrium, the nano-crystallites of heterophase fluctuations must have the same chemical potential as any other liquid region that is devoid of heterophase nucleites. Then since " $\left(\mu_{1}-\mu_{c}\right) "=0$ in Equation (2), the nucleite size (n) will diverge if $\gamma_{c l}$ is negative. This will give rise to a new colloid-like state, whence both the liquid and a crystalline state percolate the phase volume. The divergence of the cluster size $\mathrm{n}$ defines the "liquid prefreezing percolation transition" at a molecular level. Below this percolation temperature, the equilibrium thermodynamic state will be a colloidal-like mixture of pure liquid and crystalline states stabilized by a negative interfacial free energy. Compelling experimental evidence for this thermodynamic description of a pure-liquid limit above the triple point is presented in the following sections using the exemplary liquids water, and argon.

Water is the ubiquitous liquid that has a very special importance in the earth and biological sciences, but it is a wide range of unexplained properties, compared to other liquids, that make it scientifically "special" [8], with a quite remarkable, to date, range of 15 known ice polymorphs that make it uniquely different. Liquid water near freezing exhibits a plethora of 'mysterious' anomalous properties, popularized in Ball's ' $\mathrm{H}_{2} \mathrm{O}$-biography' [9]. Scientifically-characterized examples of such properties are 1) a maximum density at ambient pressure and $4^{\circ} \mathrm{C}[10] 2$ ) a long history of experimental evidence 2-state models of water [11] 3) an ability to readily supercool and by-pass the $1^{\text {st }}$-order phase transition to ice I resulting in a glass transition around $-45^{\circ} \mathrm{C}$ [12] 4) the Mpembe effect "why does hot water freeze faster than cold water" [13], and the concept of apparent "polyamorphism" [9-11] is also curious property of metastable regions of the ice-water phase diagram?

In the following sections, we will provide evidence for a line of percolation transitions on the $p, \mathrm{~T}$ plane of water that delineates the pure liquid state at temperatures above $50^{\circ} \mathrm{C}$ and for the existence of a water-ice mesophase that terminates at a glass transition around $-45^{\circ} \mathrm{C}$. This pre-freezing percolation transition to a colloid-like state, in which both water and one or more states of ice percolate the phase volume, explains many anomalous properties.

We will further examine the evidence for a pre-freezing percolation transition in liquids generally, taking the simple atomic liquid argon as an example. Evidence for such an effect can be gleaned immediately for any liquid from a closer inspection of the deviation of saturated liquid vapor pressures from a law of rectilinear diameters, which applies to the temperatures of the whole coexistence envelope, for example [14]. Figure 1 reproduces the experimental coexistence pressure of liquid argon $[15,16]$.

The expanded plot of the deviation shows that around a pressure of $1 \mathrm{MPa}$ ( $\sim 10$ atmospheres), the coexisting vapor pressure liquid argon begins to deviate from a linear LRD line around $1 \mathrm{MPa}$. A closer inspection of the pressure data suggests that the deviation has two parts to it with a discontinuity in a higher derivative indicative of a percolation transition. The density at which the equilibrium liquid as the same fugacity as the vapor increases with an increased presence of heterophase fluctuations leading to percolation, and then finite mole fraction of stable crystalline regions with the uniform $\mathrm{T}, \mathrm{p}$ and and chemical potential throughout the dispersion.

\section{PERCOLATION TRANSITIONS}

The starting point for an exact statistical treatment of the molecular theory of liquids is the formally exact expression for the Gibbs energy or Helmoltz energy (A) for a classical molecular Hamiltonian. The partition function $(Z)$ can be expressed as an integral over the whole of phase space of the Boltzmann 


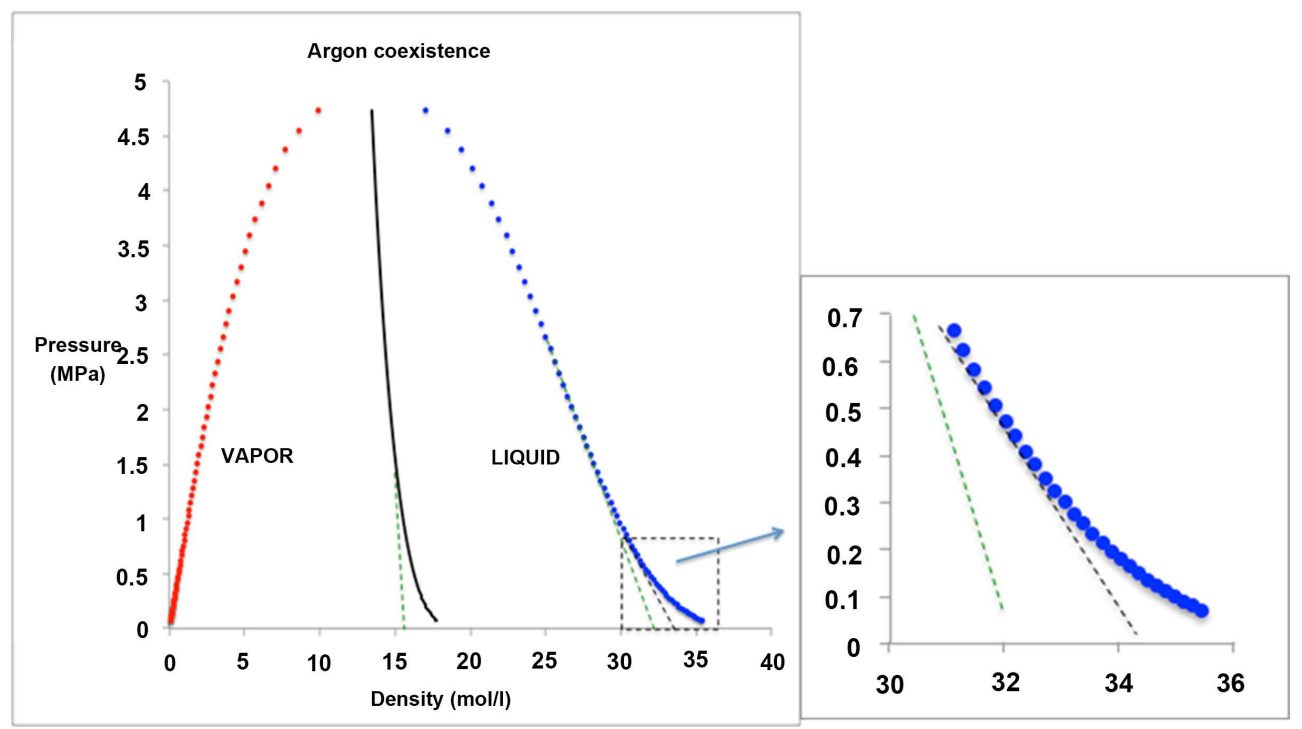

Figure 1. Saturated coexistence pressure of liquid argon as a function of density: the solid black line is the mean coexisting density defined by a law of rectilinear diameters (LRD).

probability of the entire configuration space of $N$ molecules

$$
Z(N, V, T)=Q(T) \int_{v} \cdots \int_{v} \exp \left(-\Phi\left(r_{N}\right) / k_{B} T\right) d r_{1} \cdots d r_{N}=\exp \left[-A /\left(N k_{B} T\right)\right]
$$

where $Q(T)$ is the integral over momenta space.

When there is a sizeable density gap between two different states, say of the order $10 \%$ in a typical liquid versus crystal stability criterion, it can be shown using the maximum term principle [17] that, in the thermodynamic limit that $N \rightarrow$ infinity, if a crystal phase has the higher density, the contribution to $Z$ of low-energy crystal configurations at liquid densities are negligibly rare. Likewise, the contributions of high-energy liquid configurations at crystal densities have negligible Boltzmann probability.

$Z(V, N)$ in Equation (1), and hence all the derived thermodynamic properties, however, can be expected to exhibit higher-order thermodynamic transitions of the state if the size of contributory hetero-phase clusters to the configurational integral in Equation (3), are not negligible. In this case one can envisage a divergence of heterophase contributions within an otherwise homogeneous equilibrium-state at a percolation transition. This becomes a gradual phase change, beginning and ending with higher-order phase transitions. Such a coexistence is distinctly different from a $1^{\text {st }}$-order phase transition coexistence that is characterized phenomenologically by a change in the number of degrees of freedom between oneand two-phase regions on Gibbs density surfaces. Percolation transitions are manifested as higher-order discontinuities in the Gibbs energy T-p derivatives that depend upon density and/or energy fluctuations. This implies changes of state in composition of the system, but still in keeping with Gibbs definition of a "phase", i.e. with two degrees of freedom, Tor $p$.

The change in pressure with density along an isotherm, rigidity, $\omega=(\mathrm{d} p / \mathrm{d} \rho)_{T}$ is defined as the work required increasing the density of a fluid. and is inversely related to number density fluctuations at the molecular level. Rigidity may have different but complementary origins in each coexisting state; it relates directly to the change in Gibbs energy $(\mathrm{G})$ with density at constant T according to

$$
\omega_{T}=(\mathrm{d} p / \mathrm{d} \rho)_{T}=\rho(\mathrm{d} G / \mathrm{d} \rho)_{T}
$$

Equation (4) shows that since $\mathrm{dG}$ is always $+\mathrm{ve}, \omega \geq 0$, i.e. rigidity must also always be positive: Gibbs energy cannot decrease with pressure when $T$ is constant. For both liquid and crystal states, the rigidity function must increase with density. This can be explained by the exact statistical mechanical relationship 
between the rigidity function, and fluctuations in the molecular number density $(N)$ in a thermodynamically large fixed sub-volume $(V)$ of the equilibrium fluid. An infinite number of snapshots of a dynamical system in statistical thermodynamics is a grand-canonical ensemble. From the statistical properties of this ensembleit is proven that the rigidity function is exactly related to the change in Gibbs chemical potential $(\mu)$ with number density $(N)$ according to

$$
(\mathrm{d} p / \mathrm{d} \rho)_{T}=\left(\mathrm{d} \mu / \mathrm{d} \log _{e} N\right)_{V, T}
$$

and from the thermodynamic theory of fluctuations $\left(\mathrm{d} \mu / \mathrm{d} \log _{e} N\right)_{V, T}$ is exactly and inversely proportional to an average dimensionless variance in $N$ for a constant sub-volume $V$, whereupon

$$
\left.(\mathrm{d} p / \mathrm{d} \rho)_{T}=R T /\left[<(\Delta N)^{2}\right\rangle\right]_{V, T}
$$

where $R$ is the gas constant, $T$ is the absolute temperature and $V$ is a molar volume. For the ideal gas the variance in Equation (4) is 1; it will approach zero for any solid ground state as $T \rightarrow 0 \mathrm{~K}$. The rigidity can never be negative; it varies from zero, e.g. for a near-critical supercritical fluid, to infinity for a crystalline ground state.

Second-order properties that depend upon fluctuations in energy, i.e. the heat capacities, are also expected to exhibit changes at a percolation transition since the microcrystallites, albeit with the same $T, p$ and $\mu$, as the liquid, will have a different energy. All other thermodynamic state functions derive from Equation (3), whence it can be shown that the total energy $(U)$ derivatives at constant density or volume, i.e. the isochoric heat capacity $\left(C_{v}\right)$, is related to the mean-square deviation of $\mathrm{U}$ from its average value for a Gibbs $N, V, T$ ensemble sampled from the phase integral in Equation (3).

$$
(\mathrm{d} U / \mathrm{d} T)_{v}=\left(<U^{2}>-<U>^{2}\right) /\left(k_{B} T^{2}\right)
$$

The heat capacity $C_{p}$ depends both on density and energy fluctuations. We will focus only on the two thermodynamic state functions, rigidity $\omega$ and heat capacity $C_{V}$ that depends only on energy fluctuations, to begin with.

In gas-liquid equilibria, there is a symmetry that requires the rigidities at the boundaries of existence of gas and liquid states, along supercritical isotherms to be equal [18]. There is a distribution of many small clusters in a gas with one large void, there is a distribution of many unoccupied pockets, or clusters of pockets, in the liquid with one large cluster. There can be no such crystal-liquid relationship as the rigidity of a crystal is expected behave similar to a liquid, increasing with density. However if a mesophase were to exist in which both a liquid state and a dispersed crystalline state coexisted with a negative interfacial tension, the mole fraction of either state will be a function of temperature, hence also of density. As the temperature decreases, the fraction of the crystalline state having a lower entropy, would increase, there will also be a 'latent work effect' as part of the work required to increase the density of the liquid, actually changes the composition by increasing the mole fraction of state with the higher density. For equilibrium states below the percolation transition temperature, it is conceivable that the rigidity decreases with density. This is just what is seen to occur with water (Figure 2).

\section{PREFREEZING PROPERTIES OF WATER}

Figure 2 shows that the isothermal rigidity of water decreases as density increases near to the $1^{\text {st }}$-order freezing transition water $\rightarrow$ ice I. Since, rigidity has the dimensions of energy, it can be regarded as the work required to increase the density by isothermal contraction. If as consequence of that contraction, there is also increase of density due to a change in state of part of the liquid from a lower to higher density, there will be a latent work effect, and the rigidity will decrease with density, hence also with temperature along an isobar.

This is the foremost evidence is there is a percolation transition that delineates the existence of homogeneous equilibrium liquid at a temperature above freezing; the liquid begins to freeze to a colloidal state of dispersed stable nano-crystallites that can percolate the phase volume. Since the dispersed crystallites will have the same temperature, pressure, and chemical potential as the host liquid, this is the 


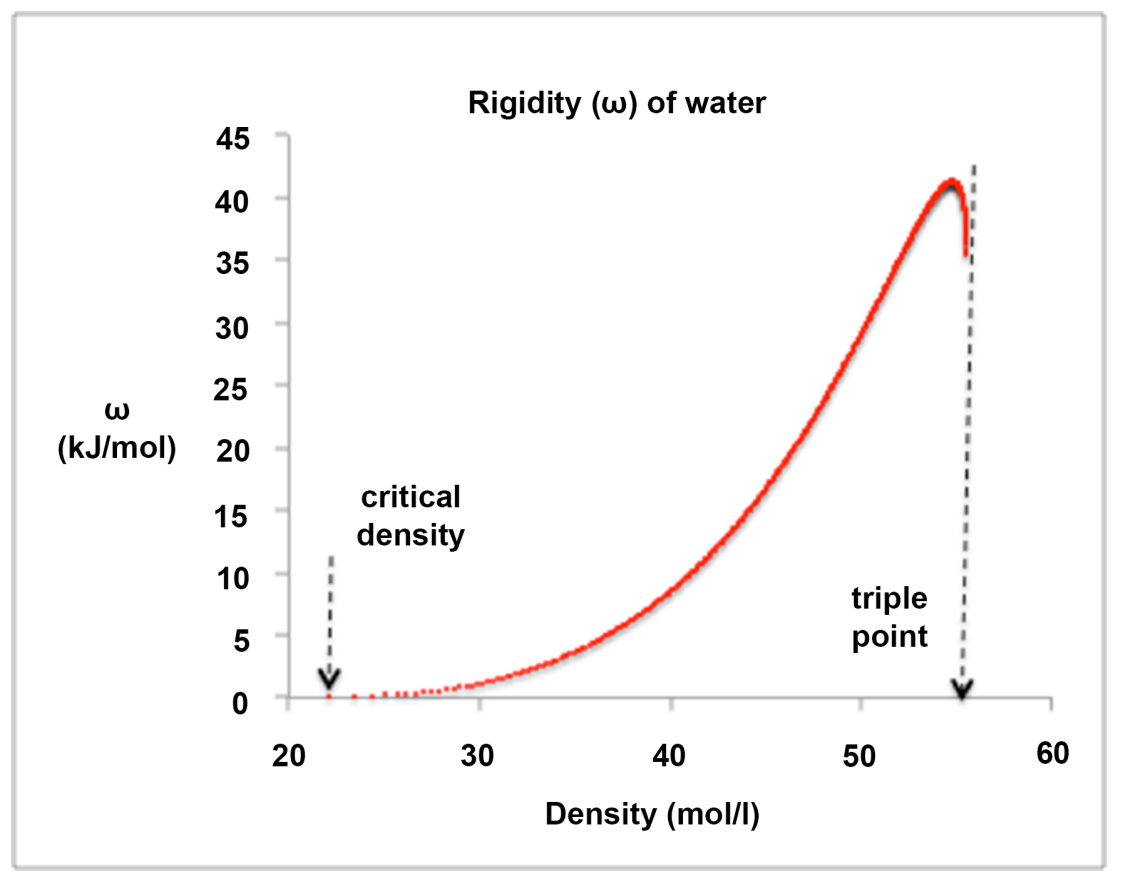

Figure 2. Rigidity of liquid water as a function of density along the equilibrium liquid-vapor coexistence line; the isothermal rigidity increases with density for liquids generally; in the case of water it takes a sudden downturn near to the triple point density (at a temperature around $50^{\circ} \mathrm{C}$ ).

equilibrium thermodynamic state above the freezing transition. As temperature decreases further, and the mole fraction of crystallites increases, so there must be both a gradual latent heat contribution to the heat capacities, as the energy decreases at constant temperature, and also a negative latent work contribution to the rigidity, as the mole fraction of the higher density crystalline state increases. The net effect of this increase in density fluctuations with mole fraction of dispersed crystallites would be a sudden downturn in rigidity as seen in water in Figure 2, at a density around that of the triple-point, but at a temperature around $50^{\circ} \mathrm{C}$, i.e. well above freezing.

If we can assume that an increase in rigidity with density defines liquid, as it does for example at supercritical temperatures we can estimate the percolation transition loci for water in the general phase diagram of the $\mathrm{p}$-T surface. The rigidity of water as given by the NIST thermophysical property compilations [16] is plotted against density in Figure 3(a) and against temperature in Figure 3(b) for the range of pressures from $0.1 \mathrm{MPa}$ (approx. 1 atmosphere) to $400 \mathrm{MPa}$ for the temperature range $0^{\circ} \mathrm{C}$ to $100^{\circ} \mathrm{C}$. Over this range of $\mathrm{T}$, water is compressed by about $10 \%$ of its ambient pressure volume.

The locus of a percolation line can also be predicted from the isochoric heat capacity data. In Figure 4 we plot the experimental $\mathrm{C}_{\mathrm{v}}$ data for 6 isobars spanning the whole liquid state range of existence, over 5 orders-of-magnitude pressure, from $0.1 \mathrm{MPa}$ to $800 \mathrm{MPa}$. Every single one of these $\mathrm{C}_{\mathrm{v}}$ isobars show a bifurcation between $50^{\circ} \mathrm{C}$ and $60^{\circ} \mathrm{C}$ being linear at the lower temperature mesophase and a non-linear deviation at the higher temperature. Below the temperature of percolation the energy fluctuations begin to decrease. Numerical estimates of combined rigidity and $\mathrm{C}_{\mathrm{v}}$ points of deviation are collected in Table 1 and plotted on a phase diagram of water (Figure 5).

The data plotted in Figure 5 for a $\omega$ - or $C_{V}$-defined percolation transition line is consistent with, and extends the observation by Maestro et al. [11]. They observed many properties of water at $1 \mathrm{~atm}$. exhibit a change in slope between around $50^{\circ} \mathrm{C}$ and $60^{\circ} \mathrm{C}$. Here we see this extends to 5 orders of magnitude pressure. These authors quote a "crossover" temperatures for thermal and electrical conductivity, dielectric constant and refractive index, proton spin-lattice relaxation time, and surface tension, and report a 


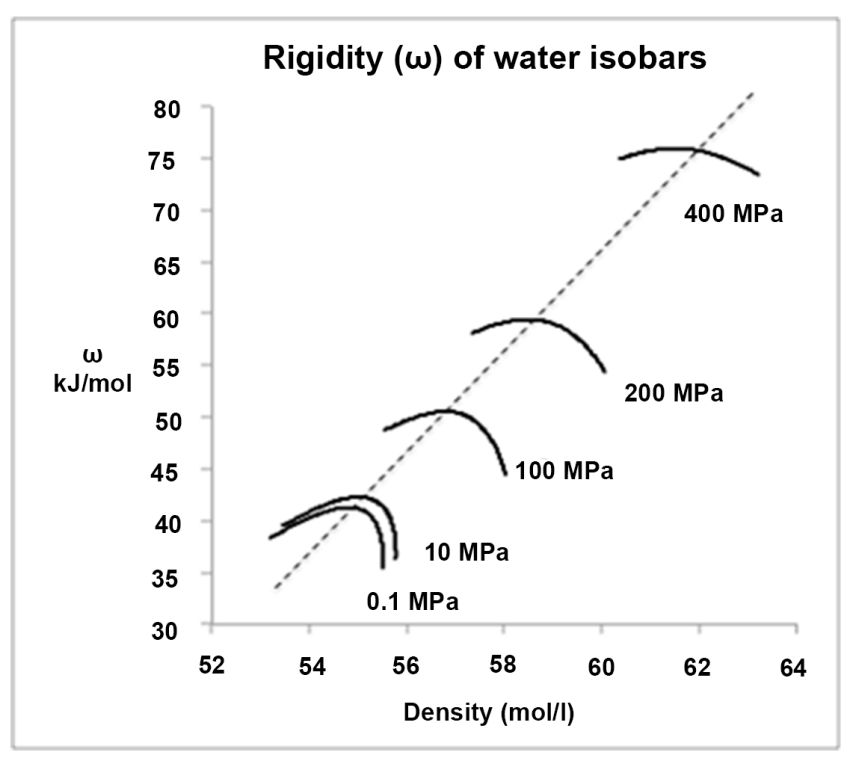

(a)

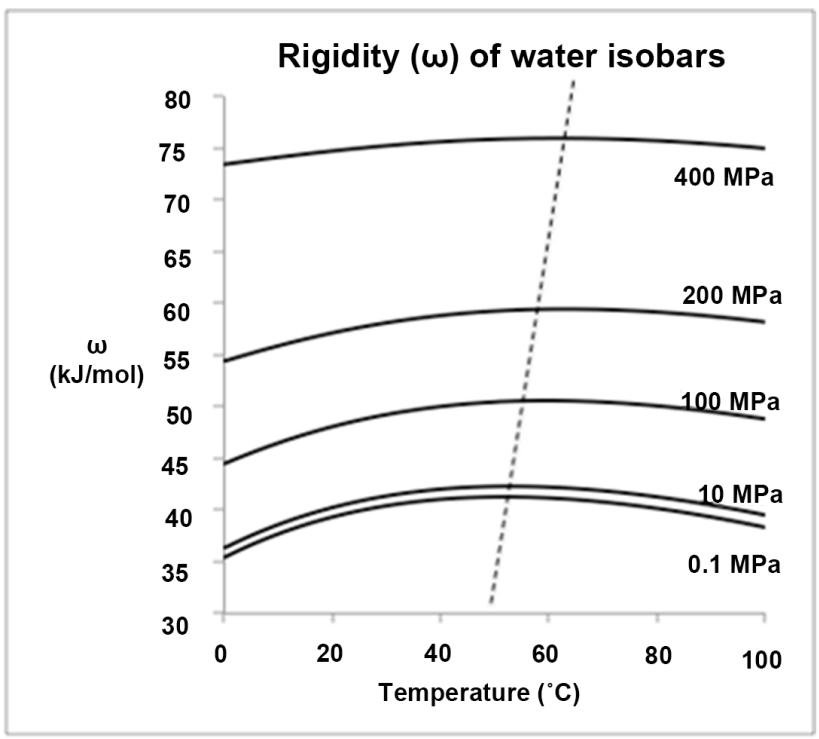

(b)

Figure 3. Rigidity of water between $0^{\circ} \mathrm{C}$ and $100^{\circ} \mathrm{C}$ along isobars from 0.1 to $400 \mathrm{MPa}$ : (a) as a function of density, (b) as a function of temperature; the dashed lines represents a loci of rigidity maxima and intercepts the coexistence line (near $0.1 \mathrm{MPa})$ at $51.2^{\circ} \mathrm{C}$.

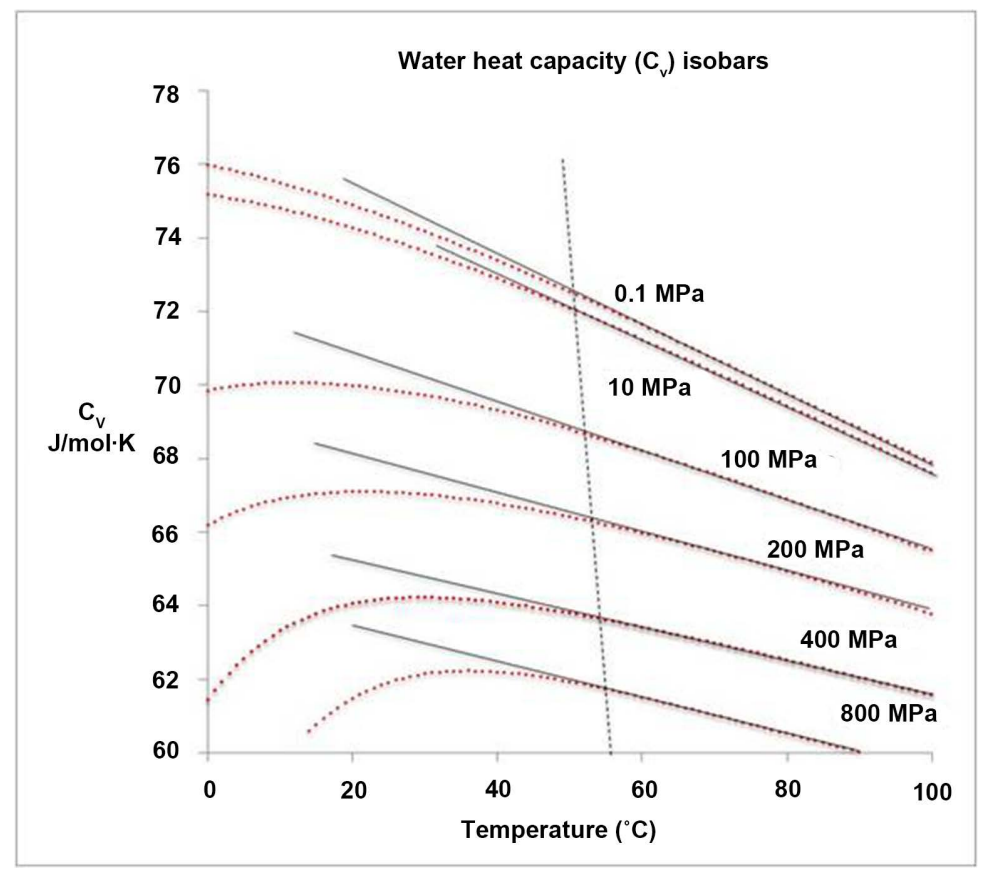

Figure 4. Isochoric hear capacity of water between $0^{\circ} \mathrm{C}$ and $100^{\circ} \mathrm{C}$ along isobars from 0.1 to $800 \mathrm{MPaas}$ a function of temperature; the inferred percolation line (dashed) intercepts the coexistence line (near $0.1 \mathrm{MPa})$ at $51.2^{\circ} \mathrm{C}$.

minimum in the piezo optical coefficient between $50^{\circ} \mathrm{C}$ and $60^{\circ} \mathrm{C}$. There is another more familiar transport property, the shear viscosity $(\eta)$ however, that may be more revealing as to the nature and composition of a mesophase below a percolation transition temperature. It can be shown that the linear shear viscosity 
Table 1. Pre-freezing percolation transition temperatures $\left(T_{p}\right)$ for water along selected isobars up to $800 \mathrm{MPa}$.

\begin{tabular}{ccc}
\hline Isobar & $T_{p}\left[\omega_{\max }\right]$ & $T_{p}\left[C_{V}\right]$ \\
$\mathrm{P}(\mathrm{MPa})$ & $(\mathrm{K})$ & $(\mathrm{K})$ \\
\hline 0.1 & 51.5 & 51.2 \\
1 & 51.2 & 51.3 \\
10 & 53.3 & 51.4 \\
100 & 59.3 & 54.0 \\
200 & 63.6 & 56.5 \\
400 & 61.6 & 58.8 \\
800 & & 55.5 \\
\hline
\end{tabular}

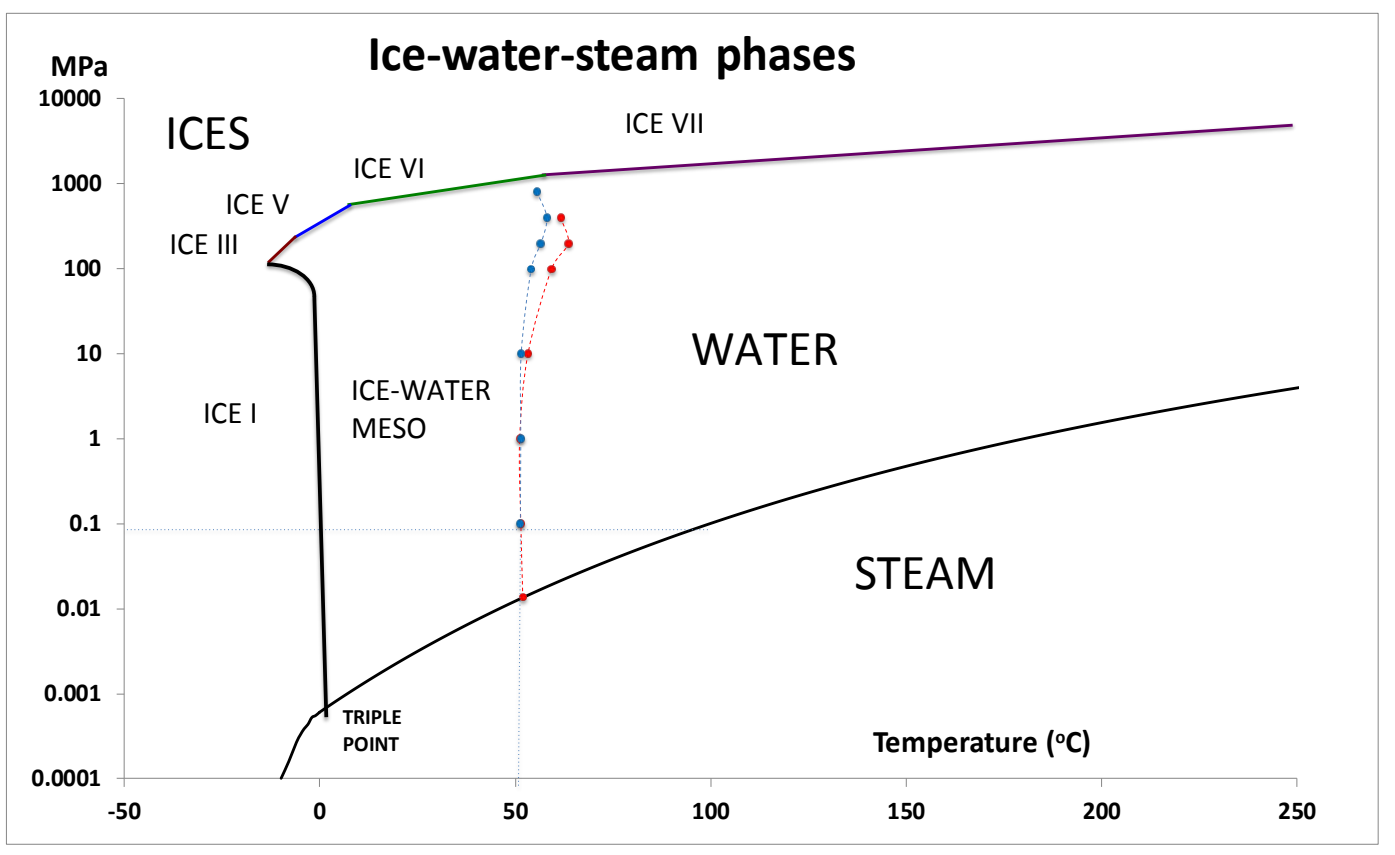

Figure 5. Phase diagram of water: the phase transition lines of stable polymorphs of ice which coexist with water at various pressures are ice I (black line) ice III (brown) ice V (blue) ice VI (green) and ice VII (purple); the present estimated prefreezing percolation line shown with the red circles of original rigidity maxima and blue circles from $\mathrm{C}_{\mathrm{v}}$ data.

coefficient also exhibits a 'crossover' in this temperature range, and that this type of behavior is consistent with a percolation transition to a colloidal dispersion of one or more ice structures in water.

The viscosity of liquids in general are well known to obey the Arrhenius activated-energy state $\left(E_{a}\right)$ model that predicts a simple exponential temperature dependence with just two parameters over a wide range of temperature at ambient pressure ( $R$ is the molar Boltzmann constant)

$$
\eta=A \exp \left(E_{a} / R T\right)
$$

A log-plot of viscosity against reciprocal temperature (Figure 6) shows that in the case of water along the 0.1 $\mathrm{MPa}$ isobar, a deviation from the Arrhenius form begins to around $50^{\circ} \mathrm{C}-60^{\circ} \mathrm{C}$. For 


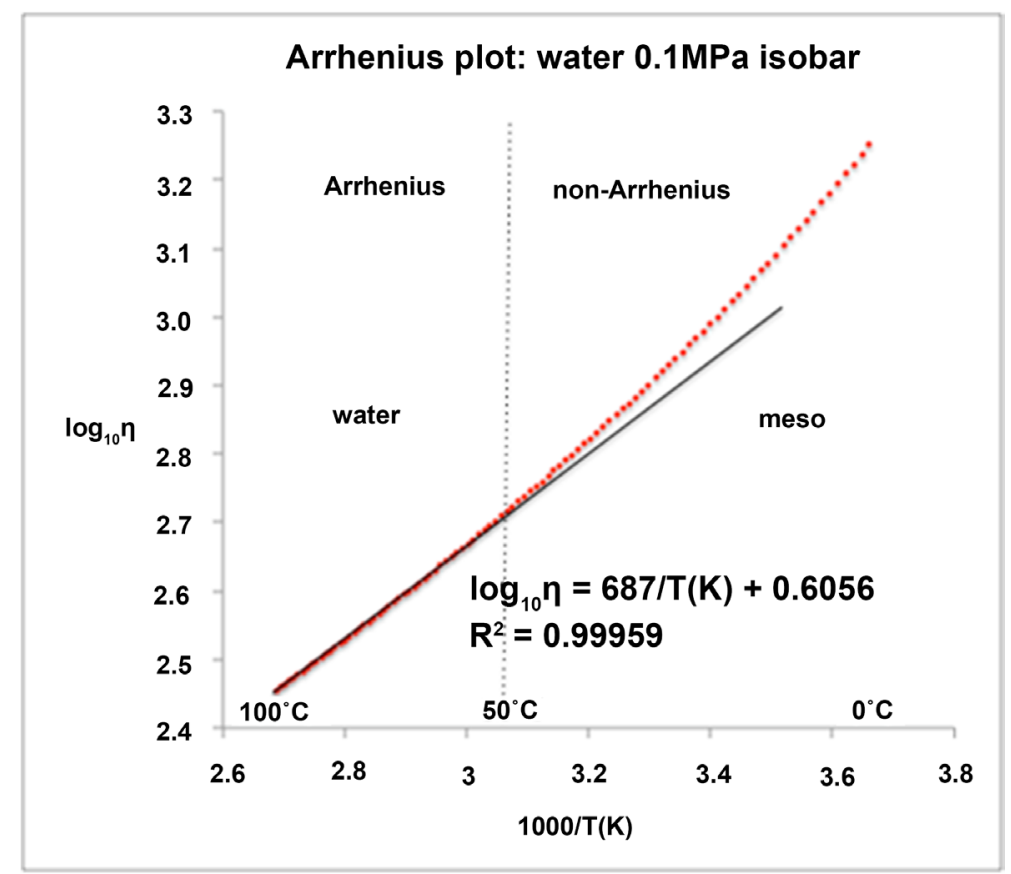

Figure 6. Viscosity of water from $0^{\circ} \mathrm{C}$ and $100^{\circ} \mathrm{C}$ plotted in the Arrhenius form along the isobar $\mathrm{p}=0.1 \mathrm{MPa}(\sim 1$ atmosphere). An EXCEL trend line is used to obtain the Arrhenius constants as shown with almost $100 \%$ linear regression in the temperature range $100^{\circ} \mathrm{C}-50^{\circ} \mathrm{C}$.

temperatures below this bifurcation the viscosity actually increases to a greater extent than is predicted by extrapolation of the Arrhenius behavior of water from above the bifurcation temperature.

This deviation in viscosity from the high-temperature Arrhenius form, is explained by the existence of a mesophase. At percolation, water becomes a colloidal dispersion of a crystalline ice in liquid-state water that can percolate the phase volume uniformly. As with 2-component colloidal dispersions, the dispersed component is stabilized by a negative surface tension at the interface between the two components. A simple formula, obtained originally by Einstein [19], for the viscosity of a dispersed solid suspended in a liquid can be used to get a rough estimate of the mole fraction of ice as the temperature decreases in the mesophase below the percolation transition.

Whilst the morphology of the dispersed ice crystalline regions is unlikely to be monodisperse spheres, a fractal distribution of shapes would have a spherical average. If the viscosity of the host water liquid below the percolation transition is denoted by $\eta_{W}$ then the viscosity of the mesophase would be given by [19]

$$
\eta_{m}=\eta_{W}(1+5 y / 2)
$$

where $y$ is volume fraction of the dispersed ice. From Equations (10) and (11) we can express the deviation from Arrhenius behavior as, a non-Arrhenius viscosity $\eta_{m}-\eta_{W}$ the fraction of ice in the mesophase is then simply related to the non-Arrhenius part of the viscosity and can be calculated from the NIST data [16] and plotted in Figure 7.

$$
y_{\text {ice }}=2 / 5\left(\eta_{m} / \eta_{W}-1\right)
$$

The first observation (Figure 6 and Figure 7) is that the temperature of the beginning of non-Arrhenius behavior is closer to $60^{\circ} \mathrm{C}$, whereas the percolation transition appears to begin closer to $50^{\circ} \mathrm{C}$ when we use thermodynamic properties as criteria. Maestro et al. [11] also found that some properties 


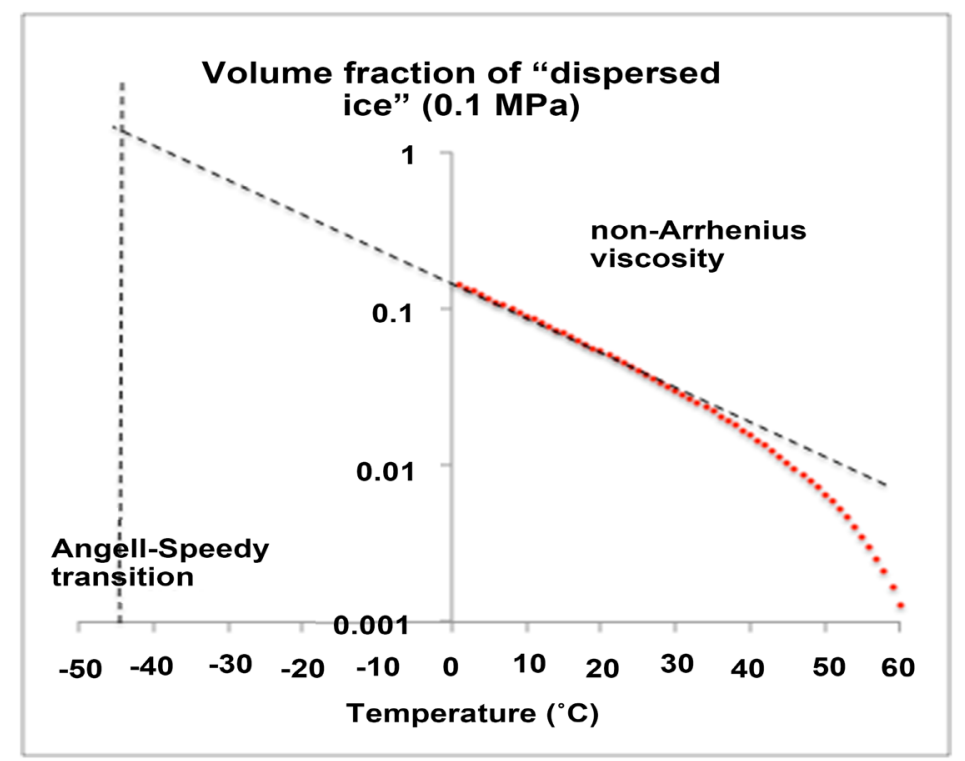

Figure 7. The volume fraction of ice in the mesophase predicted from the non-Arrhenius excess viscosity Equation (12): an extrapolation of the linear behavior of a log-plot gives a volume fraction of 1 at a temperature in the vicinity of a proposed transition [11].

begin to deviate at $60^{\circ} \mathrm{C}$ rather than $50^{\circ} \mathrm{C}$ notably transport coefficients and some optical properties. Unlike thermodynamic equilibrium properties that change at the percolation discontinuity of Gibbs energy derivatives, the transport and optical properties, however, could begin to deviate at the onset of heterophase fluctuations, i.e. at temperatures slightly above, or at pressures slightly below the percolation transition. In this region the transient embryonic crystallite fluctuations are still a part of the thermodynamic equilibrium configurations.

The predicted mole fraction of dispersed ices, which is close to the volume fraction if the densities of the dispersed ice and water are similar, is around $5 \%$ at $25^{\circ} \mathrm{C}$ and $15 \%$ at the triple point. If this behavior extrapolates into the supercooled region for temperatures below freezing, one finds a remarkable result. The volume fraction of dispersed ice is approaching unity at the temperature where there has been predicted to occur a thermodynamic phase transition resembling a second-order transition [12]. This is just what one might expect to happen when the supercooled mesophase is terminated as the mole fraction of 'liquid water' reaches zero.

\section{PREFREEZING PROPERTIES OF ARGON}

There is also evidence for a similar pre-freezing percolation transition in other liquids. It appears to be a more general phenomenon. Besides water, we have investigated the properties of rigidity and heat capacity of liquid argon. The behavior is quite different to water but there is evidence of a mesophase. Taking first the rigidity, isotherms between $90^{\circ} \mathrm{C}$ and $110^{\circ} \mathrm{C}$ were first investigated from NIST thermophysical property data [16] using the speed of sound to calculate the rigidity. Rigidity of liquid argon is not so strong a function of temperature as water, and the isotherms much close together (Figure 8). The data for the whole $90 \mathrm{~K}$ isotherm is very close to being linear over the short equilibrium range of pressure that it exists, but all the other isotherms $95 \mathrm{~K}$ to $110 \mathrm{~K}$ show a linear region at higher densities and a nonlinear region at lower density with a bifurcation; that could be the percolation loci as shown in Figure 8. Unlike the case with water, at no point does the rigidity decrease with density, but $\omega$ of the mesophase region is linear, and its derivative $\left(\mathrm{d}^{2} \rho / \mathrm{d} \rho^{2}\right)_{T}$ is constant. 


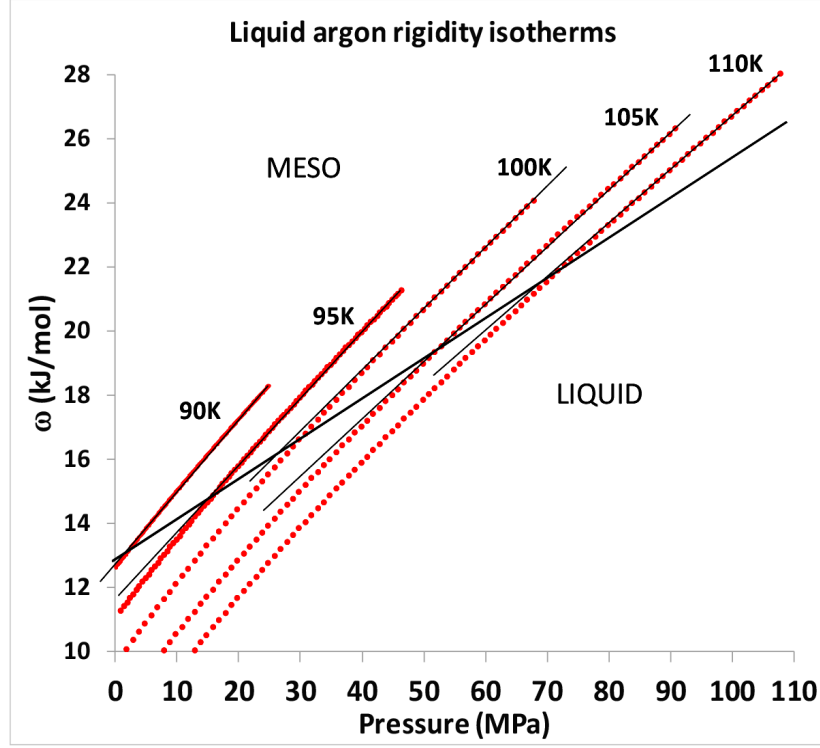

(a)

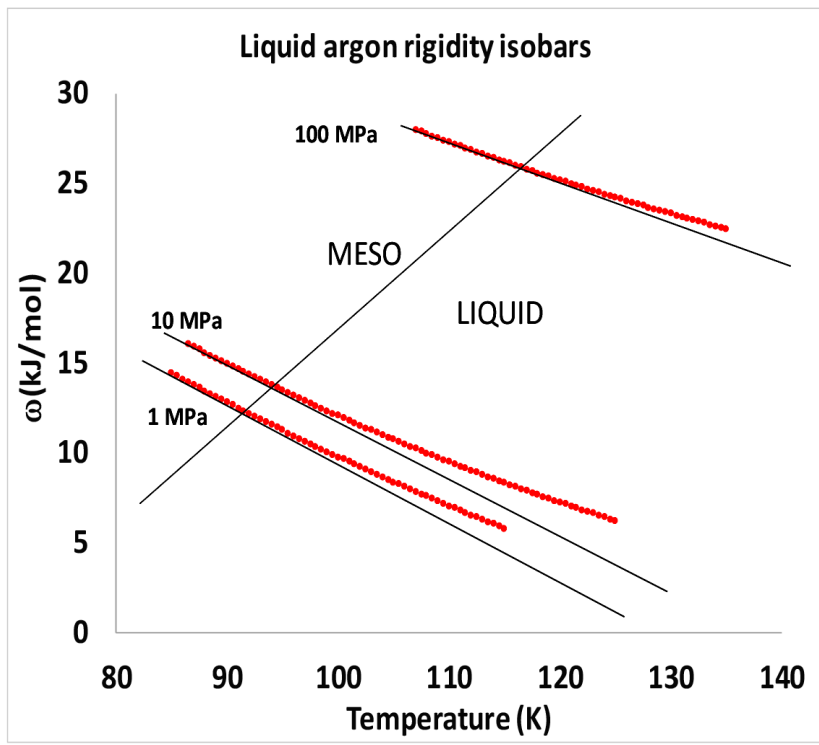

(b)

Figure 8. Experimental literature values of liquid argon rigidities shown as red data points from reference [16]; the solid lines are for guidance to show the linear region and the point of deviation; the dashed lines indicate the percolation pure liquid state limit: (a) isotherms (b) isobars.

The evidence from the isochoric heat capacity data along the same 5 isotherms is shown in Figure 9. In this case the bifurcation is more pronounced. Again, unlike the case of water, $C V(p)_{T}$ does not show a maximum, but it maintains a higher linear value than it otherwise would show a along any isotherm of the pretransition tends were to have been extrapolated from pressures above the percolation transition.

To summarize, below the percolation temperature, or above the percolation pressure, the rigidities are lower than expected, and the heat capacities are higher than expected, and for both properties everywhere in the meso-region, linear. Similar results are obtained from analysis of rigidity and heat capacity data along isobars. The points of bifurcation obtained using EXCEL trendlines of both isotherms and isobars are shown in Table 2.

The extrapolated trends of both rigidity and heat capacity can be understood, either phenomenologically if the bifurcation, or percolation transition, is deemed to be the onset of a gradual crystallization process with the mole fraction of crystalline region increasing with pressure or decreasing with temperature. If the dispersed phase has a higher density and lower energy than the pre-percolation liquid, then the rigidity is lower than the extrapolated expectation because of the latent work effect, and the heat capacity is higher than extrapolated because of the latent heat effect.

Alternatively, one can interpret the bifurcation of these two properties in terms of molecular level fluctuations in density and energy respectively. Fluctuation of both density and energy of a crystal structure structure will be significantly less than the amorphous structure with no long-range ordering. As the mole fraction of crystallite with a higher density increases, local and long-wavelength fluctuations in density and energy will both increase leading to a reduced rigidity and higher heat capacity in the mesophase, compared to the extrapolated liquid.

When the bifurcation points of Table 2 along both the isotherms and isobars of liquid argon are plotted alongside the first-order phase boundaries for argon (Figure 10), we obtain a preliminary estimate of the pre-freezing percolation transition loci for a simple liquid. At lower pressures the percolation transition point is around $6 \%$ above the triple point temperature but it increases rather sharply with pressure and appears to map onto the first-order FCC crystal-liquid equilibrium coexistence line. 


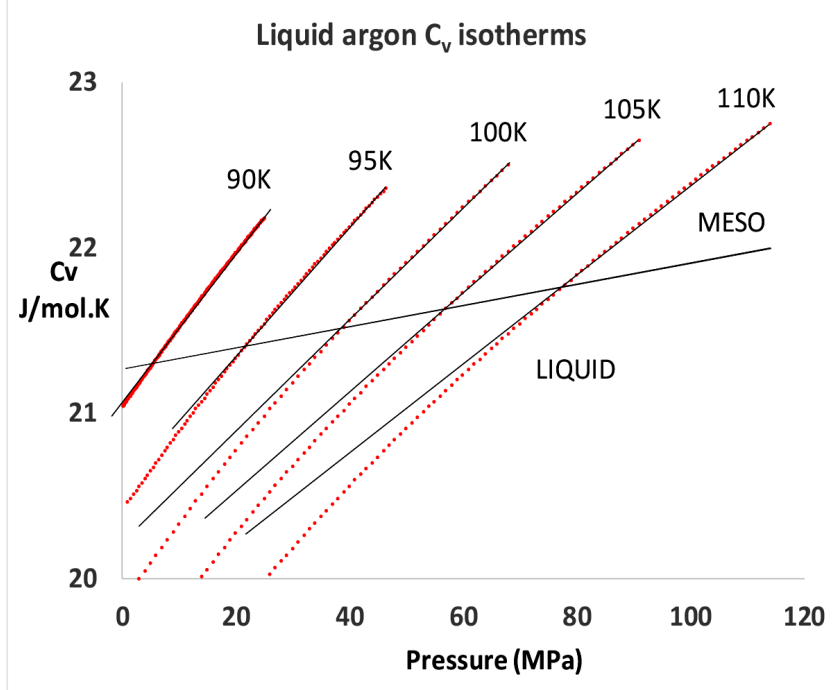

(a)

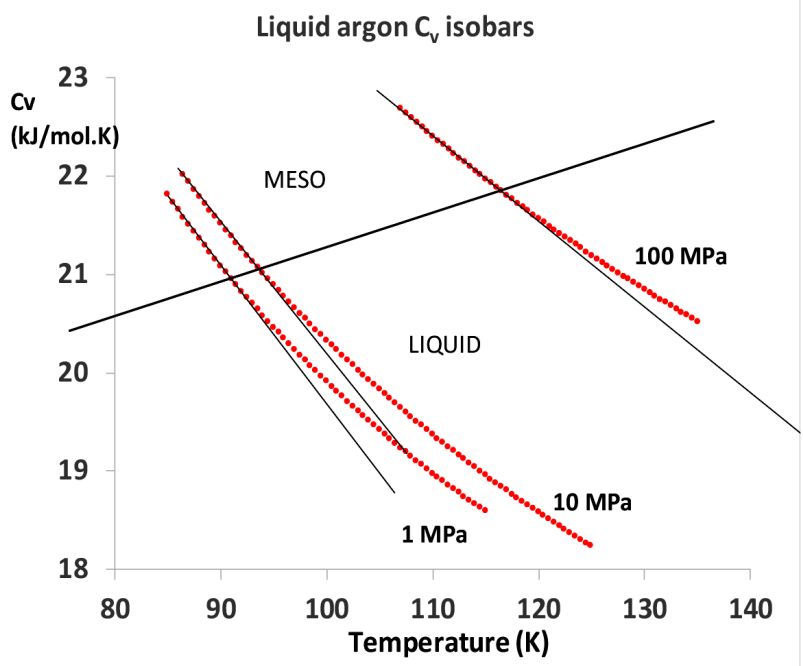

(b)

Figure 9. Isochoric heat capacities of liquid argon (red dots) using data from reference [15]; the solid line shows the linear region and the locus of points of deviation indicates percolation: (a) isotherms (b) isobars.

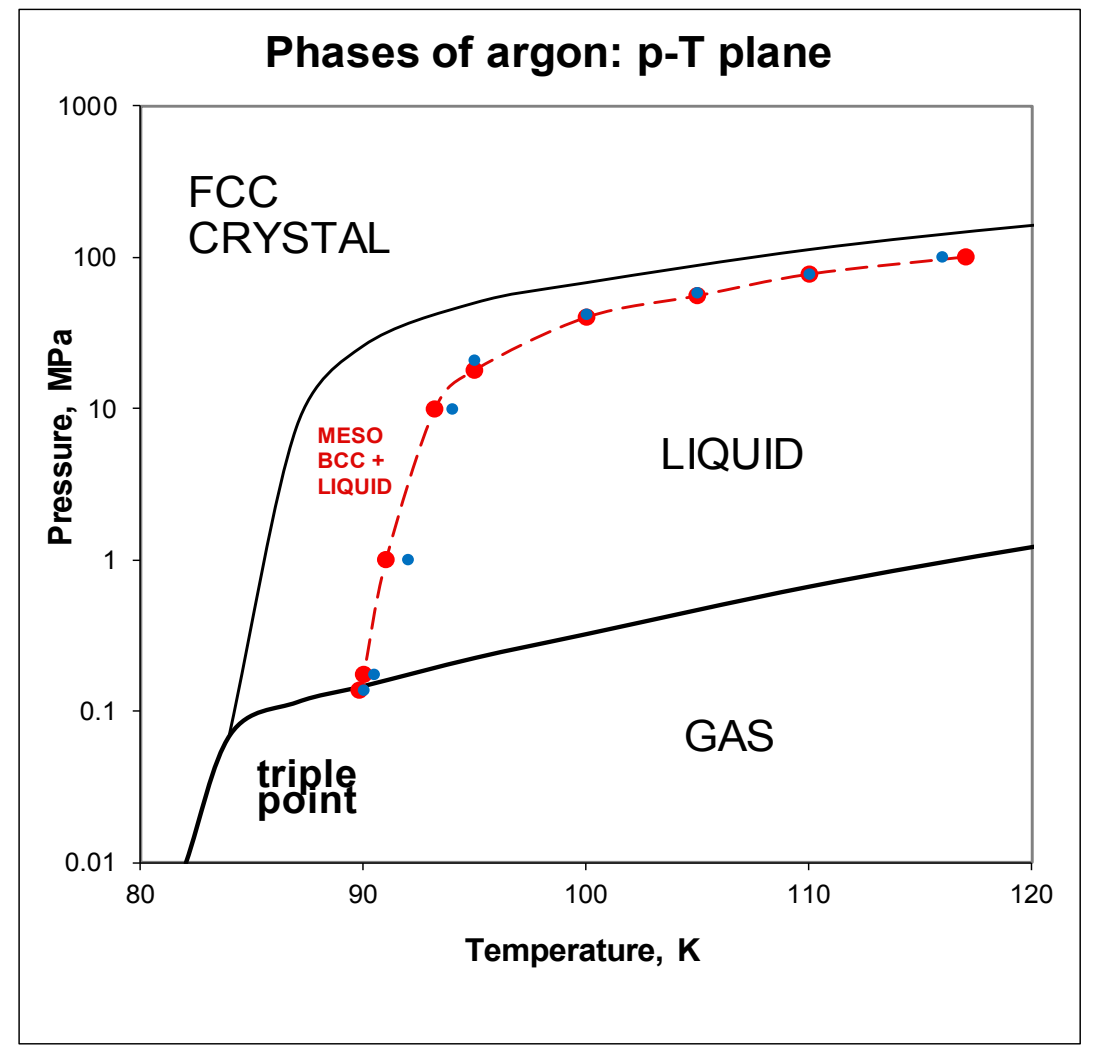

Figure 10. Phase diagram of argon: solid black lines show the first-order phase boundaries between crystal, liquid and gas phases; the red dashed line is the estimated percolation line obtained by connecting the points of discontinuity in rigidity (red dots) and heat capacity (blue dots) derivatives as described in the text. 
Table 2. Pre-freezing percolation transition pressures $\left(\mathrm{p}_{\mathrm{p}}\right)$ and temperatures $\left(T_{p}\right)$ and for liquid argon along selected isotherms and isobars respectively.

\begin{tabular}{cccccc}
\hline $\begin{array}{c}\text { Isotherm } \\
\mathrm{T}(\mathrm{K})\end{array}$ & $\mathrm{p}_{\mathrm{p}}[\omega](\mathrm{MPa})$ & $\begin{array}{c}\mathrm{p}_{\mathrm{p}}[\mathrm{Cv}] \\
(\mathrm{MPa})\end{array}$ & $\begin{array}{c}\text { Isobar } \\
\mathrm{p}(\mathrm{MPa})\end{array}$ & $\begin{array}{c}\mathrm{Tp}[\omega] \\
(\mathrm{K})\end{array}$ & $\begin{array}{c}\mathrm{T}_{\mathrm{p}}[\mathrm{Cv}] \\
(\mathrm{K})\end{array}$ \\
\hline 90 & 0.175 & 0.2 & $0.1($ coex $)$ & 89.8 & 90 \\
95 & 18 & 21.0 & 1 & 91.0 & 92 \\
100 & 40 & 42.5 & 10 & 93.2 & 94 \\
105 & 55.5 & 58.5 & 100 & 117 & 116 \\
110 & 77 & 80 & & & \\
\hline
\end{tabular}

\section{SUPERCOOLED LIQUIDS}

As a liquid is supercooled, the difference in entropy between supercooled liquid and stable crystal phase decreases because $\mathrm{C}_{\mathrm{p}}$ (liquid) $>\mathrm{C}_{\mathrm{p}}$ (crystal). By extrapolating the heat capacity of the supercooled liquid below the inevitable experimental "glass transition" temperature, it is possible to calculate a temperature at which the difference in entropies would become zero. This scenario was first observed by Kauzmann [20], and has been the subject of extensive conjecture since. If a liquid could be supercooled below its Kauzmann temperature, and it did indeed display an entropy lower than than the crystal phase, the consequences would contradict our statistical interpretation of entropy: there must be a thermodynamically self-consistent explanation [21]. The existence of a two-state meso-phase between the pre-freezing percolation transition, and the glass transition offers the ultimate resolution of this longstanding puzzle.

Kauzmann's paradox has been the subject of much debate with many different resolutions having been proposed since 1948. One resolution is to say that there must be a phase transition before the entropy of the liquid decreases. In this interpretation, the transition temperature is known as the calorimetric ideal glass transition temperature $\left(\mathrm{T}_{0}\right)$. The glass transition is not merely a kinetic effect, i.e. merely the result of fast cooling of a melt, but there is an underlying thermodynamic basis for glass formation. This is a plausible resolution but no general. thermodynamic basis has yet been proposed.

There are at least three other proposed resolutions Kauzmann paradox [see Wikipedia “Kauzmann's paradox"] 1) It could be that the heat capacity of the supercooled liquid near the Kauzmann temperature smoothly decreases to a smaller value. 2) It could be that a first order phase transition to another liquid state occurs before the Kauzmann temperature with the heat capacity of this new state being less than that obtained by extrapolation from higher temperature. 3) Kauzmann himself resolved the entropy paradox [20-22] by postulating that all supercooled liquids must crystallize before the temperature $\mathrm{T}_{0}$ is reached.

We see from Figure 11 that Kauzmann's resolution is along the right lines, but the crystallization process we find here is not quite what Kauzmann anticipated. He suggested a spontaneous homogeneous nucleation to the most stable crystal, given time; but since the kinetic relaxation time diverges, such transition could never be realized. We now see that the glass transition is in fact not that start of a spontaneous crystallization process at a singular state point, but the end of a gradual crystallization process that begins with a higher-order percolation transition above the equilibrium freezing, and ends with a second-order phase transition when there is only $100 \%$ metastable crystal polymorph(s) present. Notice also from Figure 11 the bifurcation of $\mathrm{C}_{\mathrm{p}}$ at the pre-freezing percolation transition.

This scenario was actually predicted for the glass transition hard-sphere fluid at a time when we were unaware of the metastable existence of a bcc-like phase of hard-spheres [22]. Wu and Sadus reported the discovery of a hard-sphere distorted-bcc crystal phase [23]. Moreover, the equation-of-state of the hard-sphere fluid has been shown to be nonanalytic in the prefreezing region as a consequence of heterophase fluctuations of microcrystallites in the analysis of Kolafa [24]. This new crystal structure of Wu and Sadusis quite likely to be a randomly strained bcc structure that has been termed the "hexagonal string phase"; the bcc hard-sphere crystal is unstable with respect to slip along the 1, 1, 1 diagonal. With this in 


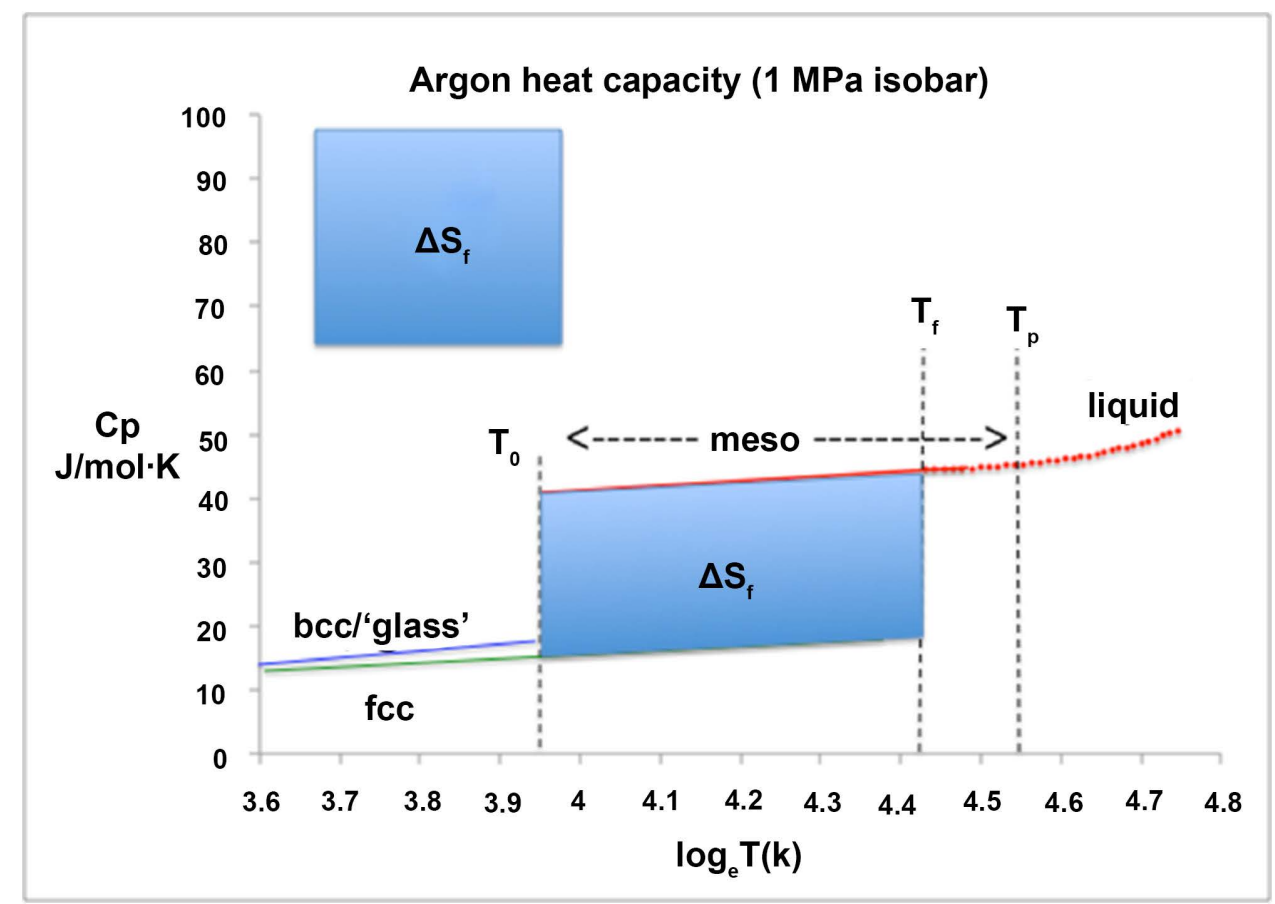

Figure 11. Typical Kauzmann heat capacity plot: argon under $1 \mathrm{MPa}$ pressure as a function of $\log _{e} T$; the square area shown is the entropy of fusion which is used to define the Kauzmann temperature $\mathrm{T}_{0}(\mathrm{~K})$ by matching it with the integrated difference between supercooled liquid and stable crystal $T<T_{f}$ heat capacities as shown; $T_{p}$ is the pre-freezing percolation transition temperature.

mind, we expect that the rheological properties of shear viscosity of supercooled simple liquids, and computer models of hard and soft spheres, could be very different from the water-ice mesophase as the dispersed states may be highly fluid "liquid-crystals" with only partial orientational ordering.

\section{CONCLUSIONS}

From the foregoing analyses, and from knowledge of the role of percolation loci in gas liquid equilibria, we can conclude that percolation transitions are now an established feature of Gibbs surfaces. These percolation loci delineate the existence of the three states of matter, gas, liquid and crystal. We find that there are stable equilibrium regions that we have termed mesophases, which are colloid-like dispersions of one state within another. It should be emphasized, however, that these mesophase regions of the $\mathrm{p}-\mathrm{V}-\mathrm{T}$ surface can belong to the same phase as a pure state, by Gibbs original definition. Because, unlike first-order two-phase coexistence regions with only one degree of freedom $\mathrm{T}$ or $\mathrm{p}$, the mesophase has two degrees of freedom in the specification of the thermodynamic state functions, i.e. both $\mathrm{T}$ and $\mathrm{p}$.

The status of the state functions within the prefreezing mesophase is that of thermodynamic equilibrium. There can be no possibility of metastability. Whereas first-order phase transitions on Gibbs density surfaces may be characterized by a metastable existence of either state on other side of the phase transition point of equal chemical potential, there can be no metastability across a second- or higher-order phase transition. The reason being since $\mathrm{d} \mu / \mathrm{d} p$ is the same for both states at the point of the transition, the state with the higher rigidity $\mathrm{d}^{2} \mu / \mathrm{d} p^{2}$ would have a lower chemical potential, and hence be the more stable, on both sides of the transition on the Gibbs density surface. Since this is a thermodynamic impossibility, the prefreezing percolation transitions in Figure 8 and Figure 10 must be the low-temperature limit of a pure 
liquid state.

Whilst the present analysis may answer some outstanding questions regarding the properties of near freezing water, and supercooled liquids more generally, there remains further unanswered questions about the nature of the colloidal and interfacial properties of the colloidal mesophase. We have found that there is a fundamental difference between cold water, below around $50^{\circ} \mathrm{C}$, and hot water. The reason for this difference is the existence of a colloidal prefreezing mesophase. Its existence can be used to explain some of the many anomalous properties of water [8-12] which will probably be a rich topic of future research for detailed explanations. Here we will conclude with just one example, the prefreezing colloidal mesophase can account for the Mpemba effect [13].

Static water under gravity in coexistence with a cold air thermostat, say $-10^{\circ} \mathrm{C}$, freezes to ice I beginning at the surface of the water. Cold water, say at $15^{\circ} \mathrm{C}$, is a colloidal dispersion of one or more of the denser ices, assuming ice VI for now. The volume fraction of ice VI in the macroscopically homogeneous bulk mesophase is around 10\% (Figure 7), but it will almost certainly be much higher at an open surface with air, perhaps $100 \%$. Therefore when cold water is cooled there can be no mechanism for nucleation to ice I from ice VI either at the surface or deep into the bulk even by homogeneous nucleation (Equation (2)), i.e. until the bulk temperature is well below $\mathrm{T}_{\mathrm{f}}$, now considering hot water, say $55^{\circ} \mathrm{C}$, instead. There will still probably be an ordered surface layer but it is more likely to resemble ice $\mathrm{I}$, at the lower density. The higher vapor pressure will cause a much more rapid cooling by evaporation effect at the surface, accompanied by a large nanoscale surface temperature gradient. This can enable the most stable ice I nucleation immediately the surface layers reach $0^{\circ} \mathrm{C}$. The heat and mass transfers for crystallization to ice I must also be faster within the crystal growth surface region of the warm water with its higher temperature gradient.

\section{ACKNOWLEDGEMENTS}

This research was presented in part at the NIST $20^{\text {th }}$ Symposium on Thermophysical Properties (June 2018). We wish to thank my colleagues there, Drs. John Maguire and Frank van Swol of Scientific Simulation Systems Inc., for many helpful discussions and positive contributions on the role of percolation transitions in fluid phase equilibria. We also thank Prof. John Finney (UCL London) for sending me a constructive critical review of the first manuscript with helpful commentary, and also another PCCP (Cambridge) reviewer, who reported this should not be published just on the basis that it is speculative. Speculation, however, is surely where all research at the forefront of understanding begins! We are therefore deeply grateful to the editorial team of Natural Science, that is now becoming the foremost international breakthrough-science journal as a consequence of their compliance with COPE peer-review ethical principles, and lack of bigotry in the peer-review process, and for their financial generosity in publishing this, and also previous related articles (references 2 and 3), with open access at little or no charge.

\section{REFERENCES}

1. Woodcock, L.V. (2011) Thermodynamic Description of Liquid-State Limits. The Journal of Physical Chemistry $B, 116,3734$.

2. Woodcock, L.V. (2013) Fluid Phases of Argon: A Debate on the Absence of van der Waals' Critical Point. Natural Science, 5, 194-206. https://doi.org/10.4236/ns.2013.52030

3. Woodcock, L.V. (2014) Gibbs Density Surface of Water and Steam: 2nd Debate on the Absence of van der Waals' Critical Point. Natural Science, 6, 411-432. https://doi.org/10.4236/ns.2014.66041

4. Yukalov, V.I. (1991) Phase Transitions and Heterophase Fluctuations. Physics Reports, 208, 395-489. https://doi.org/10.1016/0370-1573(91)90074-V

5. Bakai, A.S. (2015) Heterophase Fluctuations at the Gas-Liquid Phase Transition. LANL arxiv. 1508.04956 https://arxiv.org/cond-mat/1508.04956v1 
6. Ostwald, W. (1897) Studienüber die bildung und umwandlung fester körper. 1. Abhandlung: Ubersättigung und uberkaltung. ZeitschriftfürPhysikalischeChemie, 22, 289-330.

7. van Santen, R. (1979) The Ostwald Step Rule. The Journal of Physical Chemistry, 88, 24.

8. Finney, J.L. (2004) Water: What's So Special about It? Philosophical Transactions of the Royal Society B, 359, 1145-1165. https://doi.org/10.1098/rstb.2004.1495

9. Ball, P. (2008) Water: An Enduring Mystery. Nature, 452, 291-292. https://doi.org/10.1038/452291a

10. Gallo, P., Amann-Winkel, K., Angell, C.A., Anisimov, M.A., Caupin, F., Chakravarty, C., Lascaris, E., Loerting, T., Panagiotopoulos, A.Z., Russo, J., Sellberg, J.A., Stanley, H.E., Tanaka, H., Vega, C., Xu, L. and Pettersson, L.G.M. (2016) Water: A Tale of Two Liquids. Chemical Reviews, 116, 7463-7500. https://doi.org/10.1021/acs.chemrev.5b00750

11. Maestro, L.M., Marqués, M.I., Camarillo, E., Jaque, D., García Solé, J., Gonzalo, J.A., Jaque, F., del Valle, J.C., Mallamace, F. and Stanley, H.E. (2016) On the Existence of Two States in Liquid Water: Impact on Biological and Nanoscopic Systems. International Journal of Nanotechnology, 13, 667-677. https://doi.org/10.1504/IJNT.2016.079670

12. Speedy, R.J. and Angell, C.A. (1976) Isothermal Compressibility of Supercooled Water and Evidence for a Thermodynamic Singularity at $-45^{\circ} \mathrm{C}$. The Journal of Chemical Physics, 65, 851-858. https://doi.org/10.1063/1.433153

13. Mpemba, E.B. and Osborne, D.G. (1979) The Mpemba Effect. Physics Education, 14, 410-412.

14. Reif-Acherman, S. (2010) History of the Law of Rectilinear Diameters. Quimica Nova, 33, 2003-2013. https://doi.org/10.1590/S0100-40422010000900033

15. Gilgen, R., Kleinrahm, R. and Wagner, W. (1994) Measurement and Correlation of the Pressure Density Temperature Relation of Argon. The Journal of Chemical Thermodynamics, 26, 383-413. https://doi.org/10.1006/jcht.1994.1048

16. NIST (2018) Thermophysical Properties of Fluid Systems. http://webbook.nist.gov/chemistry/fluid/

17. Hill, T.L. (1960) Introduction to Statistical Thermodynamics. Chapter 1, Addison-Wesley Inc., Boston, 10-12.

18. Woodcock, L.V. (2016) Thermodynamics of Gas-Liquid Criticality: Rigidity Symmetry on Gibbs Density Surface. International Journal of Thermophysics, 37, 224-233. https://doi.org/10.1007/s10765-015-2031-z

19. Einstein, A. (1956) Investigations on the Theory of the Brownian Movement. Dover Publications Inc., New York.

20. Kauzmann, W. (1948) The Nature of the Glassy State and the Behavior of Liquids at Low Temperatures. Chemical Reviews, 43, 219-256. https://doi.org/10.1021/cr60135a002

21. Angell, C.A. (1970) The Data Gap in Solution Chemistry: The Ideal Glass Transition Puzzle. Journal of Chemical Education, 47, 583. https://doi.org/10.1021/ed047p583

22. Woodcock, L.V. (1981) Glass Transition in the Hard-Sphere Model and Kauzmann's Paradox. Annals of the New York Academy of Science, 371, 274-300.

23. Wu, G.-W. and Sadus, R.J. (2004) New Phase for One-Component Hard Spheres. The Journal of Chemical Physics, 120, 11686-11691.

24. Kolafa, J. (2006) Nonanalytical Equation-of-State of the Hard-Sphere Fluid. Physical Chemistry Chemical Physics, 8, 464-468. https://doi.org/10.1039/B511999E 